\title{
Náuseas e Vômitos no Pós-Operatório: Uma Revisão do "Pequeno-Grande" Problema *
}

\section{Postoperative Nausea and Vomiting: A Review of the "Minor-Major" Problem}

\author{
Neusa Lages ${ }^{1}$, Cristina Fonseca ${ }^{1}$, Aida Neves ${ }^{2}$, Nuno Landeiro ${ }^{2}$, Fernando José Abelha ${ }^{2}$
}

\section{RESUMO}

Lages N, Fonseca C, Neves A, Landeiro N, Abelha FJ - Náuseas e Vômitos no Pós-Operatório: Uma Revisão do "Pequeno-Grande" Problema

JUSTIFICATIVA E OBJETIVOS: Apesar da investigação contínua e do desenvolvimento de novos fármacos e técnicas, as náuseas e vômitos no pós-operatório (NVPO) são freqüentes e podem contribuir para o desenvolvimento de complicações com conseqüente aumento dos custos hospitalares e dos recursos humanos. Os objetivos deste artigo são a revisão dos mecanismos fisiológicos, dos fatores de risco e das medidas terapêuticas disponíveis para o manuseio de NVPO.

CONTEÚDO: Várias são as estratégias de manuseio de NVPO sugeridas neste artigo, destacando-se, no entanto, as linhas de orientação emitidas por Gan em 2003. Estas constituem a contribuição mais recente para a estratificação de risco, prevenção e tratamento dos pacientes com NVPO.

CONCLUSÕES: Embora o manuseio de NVPO tenha melhorado nos últimos anos, estes ainda ocorrem freqüentemente em grupos de risco elevado. A estratégia atual para a prevenção e manuseio de NVPO permanece por estabelecer e as linhas de orientação de Gan deverão ser adaptadas a cada população de pacientes e à instituição hospitalar.

Unitermos: ANTIEMÉTICOS; COMPLICAÇÕES: náusea, vômito pós-operatório

\section{SUMMARY}

Lages N, Fonseca C, Neves A, Landeiro N, Abelha FJ - Postoperative Nausea and Vomiting: A Review of the "Minor-Major" Problem

BACKGROUND AND OBJECTIVES: Notwithstanding continuous investigations and the development of new drugs and techniques, postoperative nausea and vomiting (PONV) are frequent and may contribute to the development of complications, thus increasing hospital and human costs. This article aimed at reviewing physiological mechanisms, risk factors and therapeutic approaches available to manage PONV.

CONTENTS: Several strategies to manage PONV are suggested in this article, but stress is given to guidelines published by Gan in 2003. They are the most recent contribution for risk stratification, prevention and treatment of PONV patients.

CONCLUSIONS: Although the management of PONV has improved in recent years, it is still common among high-risk patients. Current strategy to prevent and treat PONV is not yet established and Gan guidelines should be adapted to each population and institution.

Key Words: ANTIEMETICS; COMPLICATIONS: postoperative nausea, vomiting

\section{INTRODUÇÃO}

A s náuseas e os vômitos pós-operatórios (NVPO) são as complicações mais freqüentes na recuperação da anestesia. Até 1960, quando a utilização de agentes inalatórios como éter ou ciclopropano era freqüente, a incidência de vômitos atingia $60 \%{ }^{1}$. Hoje em dia, apesar dos avanços das

\footnotetext{
* Recebido do (Received from) Departamento de Anestesiologia e Cuidados Intensivos, Hospital São João, Porto, Portugal

1. Interno Complementar de Anestesiologia

2. Assistente Hospitalar Graduado
}

Apresentado (Submitted) em 15 de março de 2005

Aceito (Accepted) para publicação em 31 de maio de 2005

Endereço para correspondência (Correspondence to)

Dr. Fernando José Abelha

Hospital São João

Al. Professor Hernâni Monteiro

4100319 Porto

Portugal

E-mail: abelha@mail.telepac.pt

(c) Sociedade Brasileira de Anestesiologia, 2005

Revista Brasileira de Anestesiologia

Vol. 55, N 5, Setembro - Outubro, 2005 técnicas anestésicas, do uso de fármacos com curta duração de ação, e do desenvolvimento de novos antieméticos, a incidência global permanece em cerca de $20 \%$ a $30 \%{ }^{2}$. A população pediátrica não é poupada e crianças com mais de três anos apresentam incidência de NVPO de cerca de $40 \%{ }^{3}$. Em populações de risco, NVPO podem atingir $70 \%$ e cerca de $0,2 \%$ dos pacientes podem sofrer NVPO intratáveis ${ }^{2}$, retardando as altas hospitalares, exigindo internações não esperadas, um menor grau de satisfação do paciente e aumento nos custos hospitalares. Além das conseqüências descritas, poderão originar situações mais graves como deiscência de suturas, aspiração de vômito, pneumonia aspirativa, desidratação, alterações hidroeletrolíticas, rotura esofágica e aumento da pressão intracraniana.

Vários estudos foram efetuados no sentido de revelar quais seriam as preocupações mais importantes no período pós-operatório; Eberhart e col. avaliaram 220 pacientes e estratificaram qual dos 9 cenários propostos (dos 81 possíveis) para a unidade de recuperação seria mais desejável evitar. NVPO foi o cenário que maior número de pacientes deseja- 
riam evitar (49\%), seguido de outros cenários como dor $(27 \%)$ e ausência de sedação $(13 \%)^{5}$. Eberhart e col. concluíram que os pacientes estariam dispostos a aceitar outras complicações e mesmo custos pessoais adicionais para atenuar ou prevenir NVPO. Macario e col., num estudo semeIhante, propunham aos pacientes gastarem uma quantia hipotética de 100 dólares para evitar alguns efeitos colaterais da anestesia ${ }^{6}$. Este concluiu que os pacientes estariam dispostos a despender uma quantia superior para evitar vômitos (18 dólares) e náuseas (12 dólares), relativamente a evitar dor pós-operatória (17 dólares) ou tremor (8 dólares). Gan e col. num outro estudo também concluíram que os pacientes estavam dispostos a despender, às suas próprias custas, mais de 100 dólares por um antiemético eficaz ${ }^{7}$.

\section{DEFINIÇÕES E FISIOLOGIA NVPO}

Náuseas e vômitos não são sinônimos sendo importante distingui-los, uma vez que alguns fármacos são mais eficazes contra náuseas e outros contra vômitos ${ }^{8}$. Náusea é a sensação desagradável associada à necessidade de vomitar, enquanto que o vômito é a expulsão forçada de conteúdo gástrico.

O ato de vomitar é controlado pelo centro do vômito, localizado na formação reticular lateral na medula. Recebe aferências da zona quimiorreceptora (assoalho do $4^{\circ}$ ventrículo), aparelho vestibular, cerebelo, núcleo do trato solitário e ainda de centros corticais superiores. São vários os tipos de receptores envolvidos na transmissão de impulsos ao centro do vômito: os acetilcolínicos muscarínicos, os dopaminérgi- $\cos \left(\mathrm{D}_{2}\right)$, os histamínicos $\left(\mathrm{H}_{1}\right)$, os opióides, os serotoninérgi$\cos \left(5-\mathrm{HT}_{3}\right)$ e neurocinínicos $(\mathrm{NK}-1)^{9}$. As vias envolvidas no vômito podem ser acionadas por numerosos estímulos que se integram e ativam o centro do vômito. Outros fatores também contribuem para NVPO como a desidratação, certos odores, dor, apreensão e medo ${ }^{9}$. O mecanismo de ação dos antieméticos e o bloqueio de receptores ou estímulos já referidos estão apresentados na figura 1.

\section{IDENTIFICAÇÃO DE FATORES DE RISCO PARA NVPO}

Vários estudos foram efetuados para identificar fatores de risco de NVPO no sentido de prever quais os pacientes com maior risco para esta complicação ${ }^{2,10-12}$. Estes podem estar relacionados com o paciente, com o procedimento cirúrgico ou com a técnica anestésica.

Os fatores que se correlacionam com o paciente são sexo, idade, história prévia de NVPO, náuseas e vômitos com os meios de transporte e antecedentes de tabagismo. O sexo masculino parece ser um fator protetor para NVPO, com uma incidência de NVPO cerca de um terço inferior relativamente ao sexo feminino ${ }^{2,12}$. Aidade superior a 18 anos é considerada, em alguns estudos, protetora na incidência de NVPO, enquanto outros não o confirmam ${ }^{2,11,12}$. Alguns estudos atribuem um efeito protetor à história prévia de tabagismo ${ }^{2,11-13}$. Alguns procedimentos cirúrgicos foram, tradicionalmente, associados a uma maior incidência de NVPO (correção de estrabismo, cirurgia otorrinolaringológica, cirurgia ginecológica, cirurgia para ombro e cirurgia laparoscópica) ${ }^{12}$. Recentemente, num estudo efetuado em cerca de 5200 pacientes,

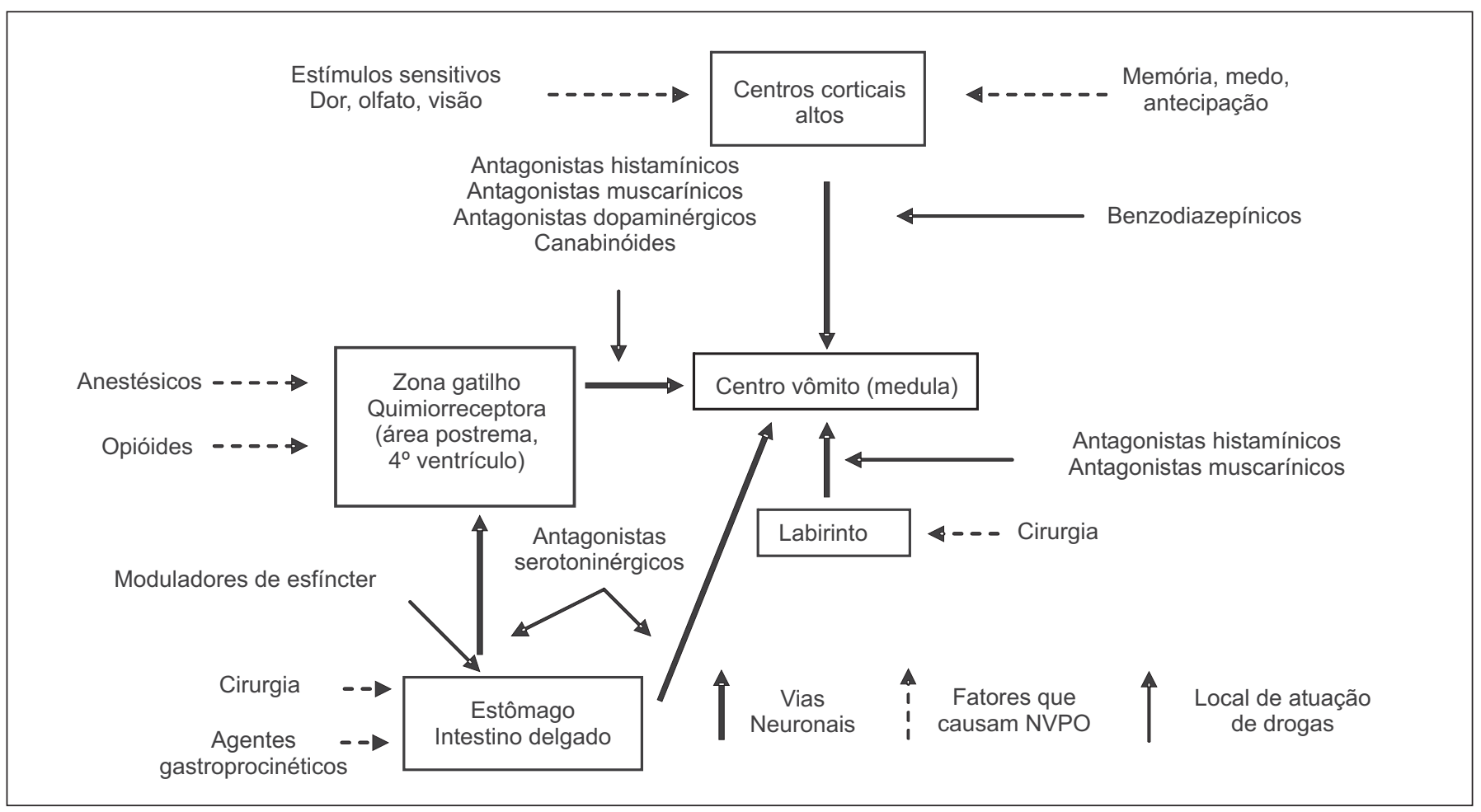

Figura 1 - Vias Neuronais e Fatores Associados com NVPO 
o risco relativo foi semelhante para todos os tipos de cirurgia, quando corrigidos para os fatores de risco de Apfel, com excepção da histerectomia e provavelmente da colecistectomia laparoscópica ${ }^{13}$. Assim os modelos preditivos de risco para NVPO que incluem o tipo de cirurgia não fornecem maior valor preditivo, quando comparados com os modelos simplificados ${ }^{14,15}$. A duração da intervenção e a experiência do cirurgião (associada com a intensidade das manipulações viscerais) são também apontadas como fatores de risco ${ }^{16}$.

Fatores relacionados com a anestesia influenciam a incidência de NVPO ${ }^{16}$. A hipotensão arterial, principalmente durante a indução, o uso de opióides no pós-operatório e a analgesia ineficaz contribuem para maior incidência de NVPO. Três metanálises demonstraram que a ausência de $\mathrm{N}_{2} \mathrm{O}$ reduz NVPO em adultos submetidos à cirurgia com risco aumentado para NVPO; o mesmo não se demonstrou em procedimentos cirúrgicos de risco reduzido ${ }^{8}$. O mecanismo proposto consiste na difusão de $\mathrm{N}_{2} \mathrm{O}$ para o ouvido médio, com estimulação do aparelho vestibular e para o intestino, com distensão deste, induzindo ativação do sistema dopaminérgico medular e aumento dos opióides endógenos no líquido cefaIorraquidiano. Outros autores advogam que a ausência de $\mathrm{N}_{2} \mathrm{O}$ se correlaciona com utilização de concentrações de oxigênio mais elevadas e que esse seria o mecanismo protetor ${ }^{17}$. O uso de anticolinesterásicos em doses elevadas, para reverter o bloqueio neuromuscular, aumenta a motilidade gástrica e NVPO ${ }^{18}$. A anestesia inalatória também acarreta risco acrescido relativamente à anestesia venosa total, principalmente no que respeito a NVPO em fase precoce ${ }^{19}$.

Tendo em conta que numerosos fatores intervêm na gênese e agravamento de NVPO, várias tentativas têm sido efetuadas para obter fatores preditivos e identificar pacientes com risco elevado para NVPO. Sinclair e col. desenvolveram um modelo matemático para prever o risco de NVPO em pacientes ambulatoriais ${ }^{12}$. Foram incluídos neste estudo prospectivo 17.638 pacientes do ambulatoriais dos quais $816(<5 \%)$ desenvolveram NVPO. Os fatores preditivos identificados para NVPO foram a idade, o sexo, a história de tabagismo, os antecedentes de NVPO, o tipo de anestesia e o tipo e a duração da cirurgia. Koivuranta e col. concluíram, num estudo prospectivo de 1107 pacientes com idade entre 4 e 86 anos, que os fatores preditivos para desenvolvimento de NVPO seriam o sexo feminino, a história prévia de NVPO, a duração da cirurgia, a história de tabagismo e a história de náuseas e vômitos com movimento ${ }^{11}$. Vários estudos têm sido efetuados, mas o modelo preditivo mais usado é o de Apfel e col. ${ }^{19}$. Este modelo baseia-se em 4 fatores de risco: sexo feminino, história de náusea e vômitos com movimento e/ou história de NVPO, ausência de tabagismo e uso pós-operatório de opióides. Aincidência NVPO seria 10\%, 21\%, 61\% e 79\%, respectivamente, se 1, 2, 3 ou 4 fatores de risco presentes (Tabela I). Os escores preditivos de Apfel são fáceis de aplicar, apresentando uma razoável preditibilidade sobre NVPO, e simultaneamente permitem a comparação entre vários grupos no domínio da investigação clinica de antieméticos. Alguns autores criticaram Apfel pela dificuldade de aplicar alguns dos seus fatores de risco ${ }^{20}$, particularmente como classificar fumantes ocasionais, como considerar pacientes sem antecedentes cirúrgicos e como prever a prescrição de opióides no pós-operatório. Apesar de todas as limitações, os escores de risco auxiliam os clínicos na definição do grupo de pacientes que se beneficiam de terapêutica profilática.

Tabela I - Escores Preditivos Segundo Apfel

\begin{tabular}{lccc}
\hline Fatores de Risco & Pontos & Riscos & Pontos \\
\hline Sexo feminino & 1 & Baixo & 0 ou 1 \\
Não fumantes & 1 & Moderado & 2 \\
$\begin{array}{l}\text { Antecedentes de náuseas e } \\
\text { vômitos com o movimento e NVPO }\end{array}$ & 1 & Elevado & 3 ou 4 \\
Uso de opióides & 1 & & \\
\hline
\end{tabular}

No início do ano de 2003, um grupo multidisciplinar de peritos reuniu-se com o objetivo de criar recomendações, baseadas na revisão exaustiva da literatura, para adultos e crianças, sobre o manuseio de NVPO e definição de grupos de risco ${ }^{21}$. O quadro I identifica os fatores risco para adultos e crianças.

Quadro I - Fatores de Risco para NVPO em Adultos e Crianças (b) (Gan e col. ${ }^{21}$ )

\begin{tabular}{|c|c|}
\hline \multicolumn{2}{|c|}{$\begin{array}{l}\text { "Consenso para o manuseio NVPO" } \\
\text { Fatores de risco para NPVO no adulto }\end{array}$} \\
\hline \multirow[t]{3}{*}{ Pacientes } & Sexo feminino \\
\hline & Ausência de tabagismo \\
\hline & $\begin{array}{l}\text { Antecedentes de NVPO ou náuseas e } \\
\text { vômitos com movimento }\end{array}$ \\
\hline \multirow[t]{3}{*}{ Anestesia } & Anestésicos inalatórios \\
\hline & $\mathrm{N}_{2} \mathrm{O}$ \\
\hline & Opióides intra e pós-operatórios \\
\hline \multirow[t]{2}{*}{ Cirurgia } & $\begin{array}{l}\text { Duração: superior a } 30 \text { minutos aumenta o } \\
\text { risco em } 60 \%\end{array}$ \\
\hline & $\begin{array}{l}\text { Tipos de cirurgias: celioscopia, ORL, } \\
\text { neurocirurgia, mama, estrabismo, } \\
\text { laparotomia, cirurgia plástica }\end{array}$ \\
\hline \multicolumn{2}{|c|}{$\begin{array}{l}\text { "Consenso para o manuseio de NVPO" } \\
\text { Fatores de risco para NPVO na criança }\end{array}$} \\
\hline \multicolumn{2}{|c|}{$\begin{array}{l}\text { Os fatores de risco em crianças são semelhantes aos dos adultos com } \\
\text { algumas particularidades: }\end{array}$} \\
\hline \multirow{2}{*}{\multicolumn{2}{|c|}{$\begin{array}{l}\text { - Os estudos em crianças limitam-se aos dados sobre vômitos e não sobre } \\
\text { náuseas; } \\
\text { - A incidência de vômitos em crianças é duas vezes superior à sua } \\
\text { incidência nos adultos; }\end{array}$}} \\
\hline & \\
\hline \multicolumn{2}{|c|}{ • A diferença em relação ao sexo não é observada em crianças; } \\
\hline \multicolumn{2}{|c|}{$\begin{array}{l}\text { - O risco aumenta de forma mais consistente com determinados tipos de } \\
\text { cirurgia. }\end{array}$} \\
\hline
\end{tabular}

\section{PROFILAXIA E TRATAMENTO NVPO}

Várias são as questões colocadas quando se aborda o tema profilaxia versus tratamento de NVPO. Devem ser tratados profilaticamente ou deve-se aguardar a sua instalação, e então, serem tratados. Se NVPO forem tratados de forma profilática, quais os fármacos ou doses devem ser utilizados. Sea opção for por uma atitude conservadora, o tratamento inicia-se com NVPO instalados, quais os fármacos ou doses 
usar? Se for realizado tratamento profilático e apesar disso o paciente apresentar NVPO, que fármacos ou doses deverão ser usados?

Com uma terapêutica profilática, muitos dos pacientes com risco reduzido para NVPO serão tratados, sem necessidade, além de se exporem aos efeitos adversos desses fármacos, enquanto outros pacientes apresentarão NVPO, apesar da profilaxia. O uso de antieméticos de rotina associa-se a custos elevados, o que numa era de contenção de custos e recursos deve ser evitado. Contudo, o uso profilático de terapêutica antiemética em pacientes com risco de moderado a elevado de NVPO apresenta relação custo-benefício eficaz e associa-se a um maior grau de satisfação dos pacientes ${ }^{22}$. Na conferência de consenso de 2003 foi recomendada profilaxia a pacientes com risco moderado a elevado para NVPO ou para aqueles com potencial morbidade associada a NVPO, especificamente deiscência de suturas, rotura esofágica, formação de hematoma e pneumonia aspirativa ${ }^{21}$. Foi recomendada a identificação de fatores de risco e a sua redução sempre que possível. Visser e col. demonstraram que o uso de propofol na indução e manutenção da anestesia reduz a incidência de NVPO precoce (primeiras 6 horas após a cirurgia) ${ }^{23}$. A incidência de NVPO diminui quando se evita o uso de anestésicos inalatórios ou óxido nitroso ${ }^{24}$. A redução de neostigmina para doses inferiores ou iguais a $2,5 \mathrm{mg}$ reduz NVPO ${ }^{18}$. Sinclair e col. demonstraram que a anestesia locorregional diminui o risco de NVPO 11 vezes relativamente à anestesia geral ${ }^{12}$. Yogendran e col. concluíram que uma fluidoterapia eficaz, com manutenção da pressão arterial durante a indução, reduz a incidência de NVPO ${ }^{25}$. O uso de frações inspiratórias de oxigênio elevadas no período peri-operatório reduz para metade a incidência de náuseas e vômitos ${ }^{17}$. Finalmente, a redução da utilização de opióides e uma analgesia eficaz (infiltração da ferida cirúrgica com anestésicos locais ou uso inibidores da ciclooxigenase) também contribuem para a redução da incidência de náuseas e vômitos ${ }^{26}$.

\section{FÁRMACOS ANTIEMÉTICOS UTILIZADOS PARA A PROFILAXIA DE NPVO (Tabela II)}

Antagonistas dos receptores da serotonina: conclui-se não existir diferença na eficácia e perfil de segurança entre os fármacos desse grupo - ondansetron, dolasetron, granisetron ou etropisetron ${ }^{21}$. Estes são mais eficazes se administrados no final da cirurgia e mais úteis na prevenção de vômitos relativamente às náuseas ${ }^{27,28}$.

Dexametasona: o mecanismo de ação dos corticóides como fármacos antieméticos permanece por esclarecer. É mais eficaz se administrada antes da indução da anestesia. A dose mais utilizada é 8 a $10 \mathrm{mg}$, por via venosa, mas doses inferiores (2,5 a $5 \mathrm{mg}$ ) também se revelaram eficazes. Apesar dos vários efeitos colaterais desses fármacos, como a infecção da ferida operatória ou supressão da glândula supra-renal, após bolus único não se revelaram quaisquer efeitos colaterais ${ }^{29}$

Droperidol: é um dos antieméticos mais estudados na profilaxia de NVPO sendo comparável na eficácia ao ondansetron ${ }^{30}$. Tem maior poder antináusea do que antivômitos e é mais eficaz quando administrado no final da cirurgia ${ }^{31}$. Quando se utilizam esquemas analgésicos com morfina, controlados pelo paciente, este parece ser o antiemético mais eficaz. As doses entre 15 e $50 \mu \mathrm{g}$ de droperidol por mg de morfina são as que apresentam melhor relação eficácia/efeitos colaterais ${ }^{32}$. Em 2001 a Food and Drug Administration emitiu um aviso sobre a associação do droperidol em pacientes com prolongamento do segmento QT no eletrocardiograma e/ou Torsade de Pointes, o que em alguns casos resultou em disritmias cardíacas e morte. Este aviso foi feito com base em 10 casos clínicos que ocorreram durante os 30 anos de uso clínico em que foram administradas doses inferiores ou iguais a $1,25 \mathrm{mg}$ deste fármaco ${ }^{33}$. Conseqüentemente o laboratório que comercializa o droperidol recomendou a mo-

\begin{tabular}{|c|c|c|c|}
\hline Administração & Fármaco & Dose Adulto & Dose Criança \\
\hline Antes da indução & Dexametasona & $\begin{array}{c}5 \text { a } 10 \mathrm{mg} \\
\text { (por via venosa) }\end{array}$ & $\begin{array}{c}150 \mu \mathrm{\mu g} \cdot \mathrm{kg}^{-1} \\
\text { (máxima } 8 \mathrm{mg} \text { ) }\end{array}$ \\
\hline \multirow[t]{5}{*}{ Após a cirurgia } & Dolasetron & $\begin{array}{c}12,5 \mathrm{mg} \\
\text { (por via venosa) }\end{array}$ & $\begin{array}{c}350 \mu \mathrm{g} \cdot \mathrm{kg}^{-1} \\
\text { (máxima } 12,5 \mathrm{mg}\end{array}$ \\
\hline & Droperidol & $\begin{array}{l}0,625 \text { a } 1,25 \mathrm{mg} \\
\text { (por via venosa) }\end{array}$ & $\begin{array}{c}50 \text { a } 75 \mu \mathrm{g} \cdot \mathrm{kg}^{-1} \\
(\text { máxima } 1,25 \mathrm{mg})\end{array}$ \\
\hline & Granisetron & $\begin{array}{l}0,35 \text { a } 1 \mathrm{mg} \\
\text { (por via venosa) }\end{array}$ & \\
\hline & Ondansetron & $\begin{array}{c}4 \text { a } 8 \mathrm{mg} \\
\text { (por via venosa) }\end{array}$ & $\begin{array}{c}50 \text { a } 100 \mu g \cdot \mathrm{kg}^{-1} \\
\text { (máxima } 4 \mathrm{mg} \text { ) }\end{array}$ \\
\hline & Tropiseton & $\begin{array}{l}5 \mathrm{mg} \\
\text { (por via venosa) }\end{array}$ & \\
\hline $\begin{array}{l}\text { Na noite anterior à cirurgia ou } \\
4 \text { horas antes do final da cirurgia }\end{array}$ & Escopolamina & Patch transdérmico & \\
\hline Sem indicação & Dimenhirinato & $\begin{array}{c}1 \text { a } 2 \text { mg. } \mathrm{kg}^{-1} \\
\text { (por via venosa) } \\
0,5 \mathrm{mg}^{-1} \mathrm{~kg}^{-1} \\
\text { (por via muscular) }\end{array}$ & $0,5 \mathrm{mg} \cdot \mathrm{kg}^{-1}$ \\
\hline
\end{tabular}


nitorização eletrocardiográfica durante e após 2 a 3 horas da sua administração e contra-indicou a sua utilização em pacientes do sexo masculino com intervalos QT superiores a 440 ms ou em pacientes do sexo feminino com intervalos QT superiores a $450 \mathrm{~ms}$. Outros pacientes de risco para a sua utilização são aqueles com insuficiência cardíaca congestiva, bradicardia, hipertrofia ventricular, hipocalemia, hipomagnesemia, pacientes medicados com diuréticos ou outros fármacos que possam originar prolongamento do intervalo QT. Alguns defensores do droperidol argumentam que não estão descritos, em revistas indexadas, casos clínicos em que se associou prolongamento do intervalo QT, disritmias cardíacas ou morte ao droperidol, quando usado nas doses apropriadas para evitar NPVO.

Outros fármacos antieméticos: a escopolamina transdérmica deve ser aplicada na noite anterior à cirurgia ou cerca de 4 horas antes do fim da cirurgia ${ }^{34}$. Não é aconselhável em idosos e tem como limitação o início de ação tardio (2 a 4 horas após a sua administração) ${ }^{35}$. As fenotiazidas (prometazinae proclorperazina) são eficazes se administradas no final da cirurgia, mas os seus efeitos adversos (sedação, tonturas, desidratação) limitam a sua utilização principalmente em regime ambulatorial ${ }^{36}$. A efedrina por via muscular parece ser eficaz, principalmente se as náuseas e os vômitos estão associados à desidratação ou hipotensão arterial ${ }^{37}$.

Técnicas não farmacológicas: a acupuntura, a compressão, a estimulação nervosa transcutânea e a hipnose mostraram-se eficazes se utilizadas antes da cirurgia ${ }^{38}$, mas carecem de maior avaliação.

Evidências insuficientes: a metoclopramida nas doses utilizadas na prática clínica (10 mg), por via venosa, não está recomendada no manuseio de NVPO ${ }^{39}$, assim como a raiz de gengibre ${ }^{40}$ ou os canabinóides ${ }^{41}$.

Nos últimos anos, tem sido defendida uma abordagem antiemética multimodal baseada no conceito multifatorial da etiologia de NVPO. Assim, utilizando-se mais que uma classe de antieméticos, atingir-se-iam mais do que um tipo de receptores envolvidos em NVPO.

$\mathrm{Na}$ conferência de consenso, já citada, é recomendada para os pacientes com risco reduzido de NVPO, ausência de profilaxia; para os de risco moderado, profilaxia com monoterapia ou terapêutica combinada; para os de risco elevado, profilaxia com dois ou três fármacos antieméticos de classes diferentes ${ }^{21}$. No entanto, um estudo recente salientou que a combinação de várias modalidades terapêuticas associa-se a maiores custos, a maior risco de efeitos colaterais e à ausência do benefício somatório dos vários fármacos antieméticos. Para estes autores a terapêutica combinada deverá ser reservada apenas para pacientes com risco elevado de NVPO ou para aqueles cujas NVPO poderão colocá-lo em risco $^{42}$. Neste grupo específico existe maior número de candidatos para profilaxia antiemética, uma vez que a incidência de NVPO duplica. Os antagonistas dos receptores $5-\mathrm{HT}_{3}$ deverão ser os fármacos profiláticos de primeira linha. O uso de droperidol, pelos seus efeitos extra piramidais, deverá ficar reservado para crianças internadas quando todas as outras terapêuticas se revelem ineficazes ${ }^{21}$.
Diretrizes para o tratamento de NVPO nos pacientes em que não foi efetuada profilaxia ou para aqueles cuja profilaxia falhou (Quadro II) ${ }^{21}$.

Quadro II - Tratamento de NVPO nos Pacientes que não foi Efetuada Profilaxia ou naqueles que esta Falhou (Gan e col. ${ }^{21}$ )

\begin{tabular}{ll} 
Se terapêutica inicial & \multicolumn{1}{c}{ Tratamento } \\
\hline $\begin{array}{l}\text { Ausência de profilaxia ou } \\
\text { dexametasona }\end{array}$ & $\begin{array}{l}\text { Doses reduzidas de } \\
\text { antagonistas dos receptores } \\
\text { serotoninérgicos }\end{array}$
\end{tabular}

Antagonistas dos receptores serotoninérgicos e $2^{\circ}$ fármaco de outra classe

Terapêutica tripla com antagonistas dos receptores serotoninérgicos e $2^{\circ}$ e $3^{\circ}$ fármaco de classes diferentes

\section{Fármaco de outra classe}

Se NVPO ocorrem $<6$ horas de pós-operatório: fármaco de classe diferente ou propofol $20 \mathrm{mg}$ (se UCPO)

Se NVPO ocorrem $\geq 6$ horas de pós-operatório: repetir antagonistas dos receptores serotoninérgicos e droperidol ou fármaco de outra classe

Em primeiro lugar devem ser excluídos fármacos ou fatores mecânicos que possam originar NVPO e só depois iniciar a terapêutica.

Pacientes em que não foi realizada profilaxia para NVPO devem ser tratados com antagonistas dos receptores $5-\mathrm{HT}_{3}$. Doses reduzidas a um quarto das doses utilizadas para profilaxia revelaram-se eficazes. Dados relativamente a doses e eficácia de outros antieméticos utilizados para tratamento de NVPO são ainda escassos. Em pacientes cuja profilaxia com dexametasona falhou, recomenda-se terapêutica com doses reduzidas de antagonistas dos receptores $5-\mathrm{HT}_{3}$.

$\mathrm{Se}$ a profilaxia com antagonistas dos receptores $5-\mathrm{HT}_{3}$ faIhou, não se devem utilizar doses subseqüentes da mesma classe de fármacos nas primeiras seis horas após cirurgia. Se a profilaxia com antagonistas receptores $5-\mathrm{HT}_{3}$ associados à dexametasona foi ineficaz, devem ser administrados antieméticos de uma classe diferente.

Na generalidade, pacientes que apresentam NVPO nas primeiras seis horas após a cirurgia deverão ser tratados com antieméticos de classes diferentes dos utilizados para profilaxia. Se NVPO surgem após seis horas da cirurgia, os fármacos usados para profilaxia poderão ser repetidos, com exceção da escopolamina ou dexametasona, pois apresentam duração de ação superior a seis horas.

O propofol em doses reduzidas pode ser utilizado desde que os pacientes se encontrem em ambiente de vigilância continua.

\section{CONCLUSÃO}

Embora o manuseio de NVPO tenha melhorado nos últimos anos, estes ainda ocorrem freqüentemente em grupos de risco elevado. 
A identificação de fatores de risco para NVPO, efetuada freqüentemente no pré-operatório beneficia a terapêutica profilática. A publicação em 2003 de recomendações internacionais para o manuseio de NVPO permitiu definir os pacientes que se beneficiam de terapêutica única, dupla ou tripla em função do nível de risco identificado.

Dos numerosos fármacos disponíveis, o droperidol, os antagonistas dos receptores $5 \mathrm{HT}_{3}$ e a dexametasona têm um lugar de destaque no controle de NVPO e a sua associação, baseada em mecanismos de ação e relação eficácia/tolerância, permitiu um controle mais eficaz de NVPO.

Apesar dos dados da literatura limitados e das variações existentes na identificação dos fatores de risco, as recomendações estabelecidas para os adultos são extrapoladas para as crianças. Deve ser relembrado que a existência destas recomendações não evita, no entanto, a análise individualizada e a sua adaptação à população, técnicas cirúrgicas e anestésicas em questão.

\section{Postoperative Nausea and Vomiting: $A$ Review of the "Minor-Major" Problem}

Neusa Lages, M.D. Cristina Fonseca, M.D.; Aida Neves, M.D.; Nuno Landeiro, M.D.; Fernando José Abelha, M.D.

\section{INTRODUCTION}

Postoperative nausea and vomiting (PONV) are among most frequent complications during anesthetic recovery. Until 1960, when inhalational anesthetics such as ether or cyclopropane were widely used, the incidence of vomiting could reach $60 \%{ }^{1}$. Today, in spite of the progress of anesthetic techniques, of short-lasting drugs and of the development of new antiemetic drugs, global incidence is still approximately $20 \%$ to $30 \%{ }^{2}$. Pediatric patients are not spared and children above 3 years of age have an incidence of PONV of approximately $40 \%{ }^{3}$. In risk populations, PONV may reach $70 \%$ and approximately $0.2 \%$ of patients may suffer untreatable PONV 2 , delaying hospital discharge, requiring unexpected admissions, generating a low level of patients' satisfaction and increasing hospital costs. In addition to above-mentioned complications, more severe situations may be generated, such as suture dehiscence, vomiting aspiration, aspiration pneumonia, dehydration, hydroelectrolytic changes, esophageal rupture and increased intracranial pressure.

Several studies were carried out to determine major postoperative concerns. Eberhart et al. have evaluated 220 patients and have stratified which of the 9 proposed scenarios (from 81 possible) for the recovery unit would be more desirable to be avoided. PONV was the scenario most patients would like to avoid ( $49 \%)$, followed by others such as pain $(27 \%)$ and no sedation $(13 \%)^{5}$. Eberhart et al. have concluded that patients would be willing to accept other complications and even additional personal costs to minimize or prevent PONV. Macario et al. in a similar study have proposed to patients to spend a hypothetical amount of 100 dollars to prevent some anesthetic side effects ${ }^{6}$. The conclusion was that patients would be willing to spend a higher amount to prevent vomiting (18 dollars) and nausea (12 dollars), as compared to preventing postoperative pain (17 dollars) or shivering (8 dollars). Gan et al., in a different study, has also concluded that patients were willing to spend more than 100 dollars, at their own expenses, for an effective antiemetic drug ${ }^{7}$.

\section{PONV DEFINITIONS AND PHYSIOLOGY}

Nausea and vomiting are not synonyms, being important to separate them since some drugs are more effective against nausea and others against vomiting ${ }^{8}$. Nausea is the uncomfortable sensation associated to the need for vomiting, while vomiting is the forced expelling of gastric content.

The act of vomiting is controlled by the vomiting center, located in the lateral reticular formation of the spinal cord. It receives afferences from the chemoreceptor zone $\left(4^{\text {th }}\right.$ ventricle floor), vestibular tract, cerebellum, solitary tract nucleus and upper cortical centers. There are several receptors involved in transmitting impulses to the vomiting center: muscarinic, dopaminergics $\left(D_{2}\right)$, hystaminergics $\left(H_{1}\right)$, opioids, serotoninergics $\left(5-\mathrm{HT}_{3}\right)$ and neurokyninics $(\mathrm{NK}-1)^{9}$. Vomiting pathways may be triggered by several stimulations that integrate and activate the vomiting center. Other factors also contribute for PONV, such as dehydration, some odors, pain, concern and fear ${ }^{9}$. Mechanisms of action of antiemetics and receptors blockade or stimulations already mentioned are represented in figure 1.

\section{IDENTIFICATION OF PONV RISK FACTORS}

Several studies have been carried out to identify PONV risk factors and anticipate patients with higher risk of this complication $^{2,10-12}$. These may be related to patients, surgical procedure or anesthetic technique.

Patient-related factors are gender, age, previous PONV history, motion sickness and smoking. Males seem to be more protected against PONV, with one third lower incidence as compared to females ${ }^{2,12}$. Age above 18 years is considered by some studies as protective against PONV, while others do not confirm it $2,11,12$.

Some studies attribute a protecting effect to previous smoking history ${ }^{2,11-13}$. Some surgical procedures are traditionally associated to higher incidence of PONV (strabismus correction, ENT surgery, gynecologic surgery, shoulder surgery and laparoscopic surgery) ${ }^{12}$. In a recent study with approximately 5200 patients, the relative risk was similar for all types of surgery, when corrected for Apfel's risk factors, except for hysterectomies and possibly laparoscopic cholecystectomy ${ }^{13}$. So, predictive models of PONV risk including surgery type do no add to predictive value as compared to simplified models ${ }^{14,15}$. Surgery length and surgeon's experiences (associated to the intensity of visceral manipulation) are also considered risk factors ${ }^{16}$.

Revista Brasileira de Anestesiologia Vol. 55, N 5 , Setembro - Outubro, 2005 


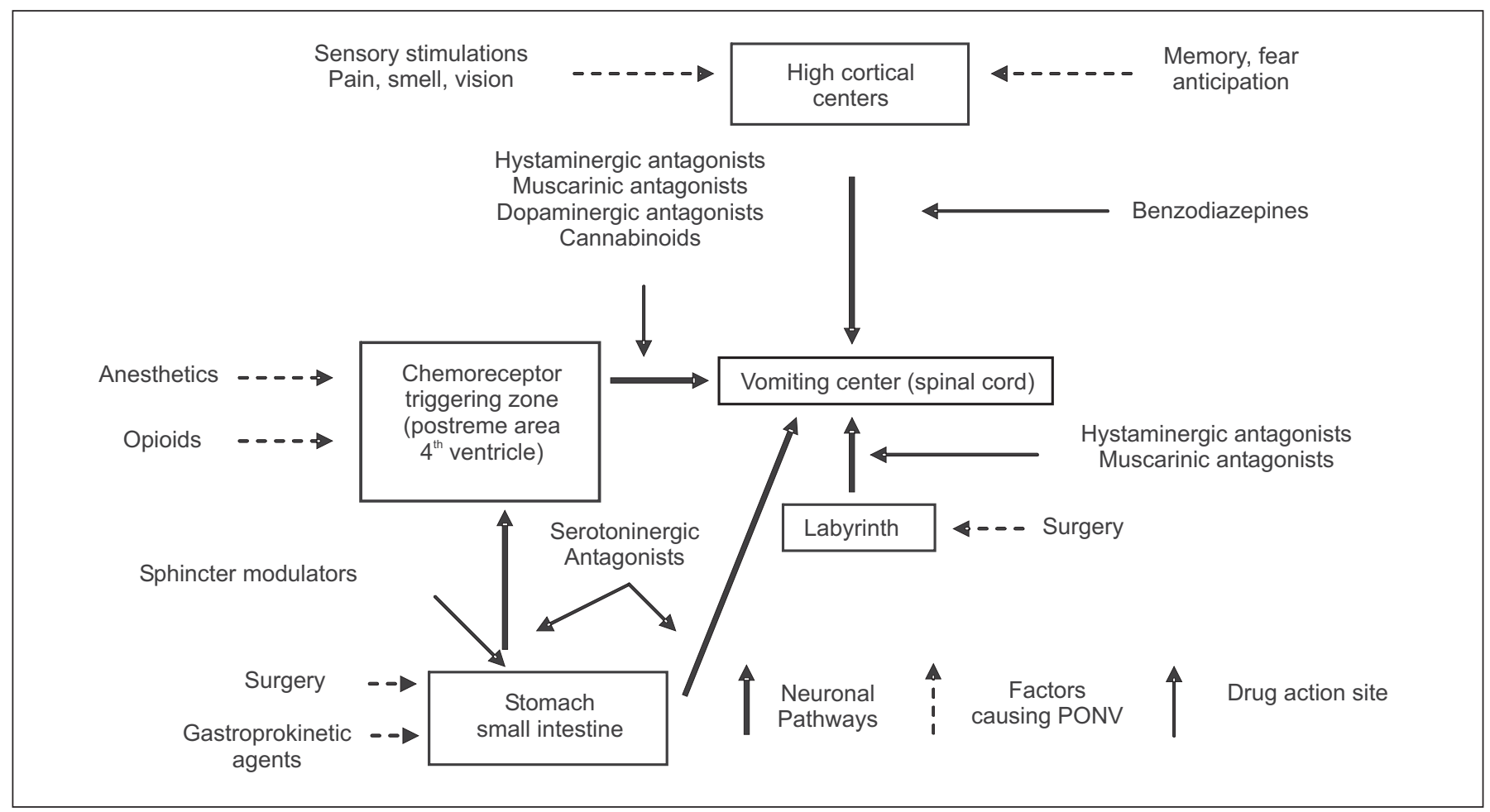

Figure 1 - Neuronal Pathways and Factors Associated to PONV

Anesthesia-related factors influence the incidence of PONV

${ }^{16}$. Hypotension, especially during induction, postoperative opioids and ineffective analgesia contribute to a higher incidence of PONV. Three meta-analysis have shown that lack of $\mathrm{N}_{2} \mathrm{O}$ decreases PONV in adults submitted to low risk procedures ${ }^{8}$. Proposed mechanism consists of $\mathrm{N}_{2} \mathrm{O}$ spread to middle ear with vestibular tract stimulation, and to the bowel distending it, inducing spinal cord dopaminergic system activation and increasing endogenous opioids in CSF. Other authors advocate that lack of $\mathrm{N}_{2} \mathrm{O}$ is correlated to the use of higher oxygen concentrations and that this would be the protecting mechanism ${ }^{17}$. High doses of anticholinesterase drugs used to antagonize neuromuscular block increase gastric motility and PONV ${ }^{18}$. Inhalational anesthesia is also of higher risk as compared to total intravenous anesthesia, especially in terms of early PONV ${ }^{19}$.

Considering that several factors influence PONV genesis and worsening, many attempts have been made to obtain predictive factors and identify patients at high risk for PONV. Sinclair et al. have developed a mathematical model to anticipate PONV risk in ambulatory patients ${ }^{12}$. Participated in this prospective study 17,638 ambulatory patients of whom 816 $(<5 \%)$ have developed PONV. Predictive factors identified were: age, gender, and history of smoking, history of PONV, type of anesthesia and surgery type and length. Koivuranta et al. have concluded in a prospective study with 1107 patients aged 4 to 86 years, that predictive factors for PONV would be female gender, previous PONV history, surgery length, history of smoking and history of motion sickeness ${ }^{11}$.
There are several studies on the subject, but the most popular predictive model is the model developed by Apfel et al. ${ }^{19}$. This model is based on 4 risk factors: female gender, history of motion sickness and/or history of PONV, no smoking and postoperative opioids. The incidence of PONV would be $10 \%, 21 \%, 61 \%$ and $79 \%$, respectively, whether $1,2,3$ or 4 factors are present (Table I).

Table I - Predictive Scores According to Apfel

\begin{tabular}{lccc}
\hline Risk factors & Points & Risks & Points \\
\hline Female gender & 1 & Low & 0 or 1 \\
Non smokers & 1 & Moderate & 2 \\
History of motion sickness & 1 & High & 3 or 4 \\
and PONV & 1 & & \\
Use of opioids & & & \\
\hline
\end{tabular}

Apfel's predictive scores are easy to apply, with reasonable predictability of PONV; in addition, they allow for the comparison among several groups in the domain of clinical antiemetic investigations. Some authors have criticized Apfel for the difficulty in applying some of his risk factors ${ }^{20}$, especially how to classify occasional smokers, how to consider patients without surgical history and how to anticipate the prescription of postoperative opioids. In spite of all limitations, risk scores help clinicians define the group of patients benefiting of preventive therapy.

In the early 2003, a multidisciplinary group of experts has met to create recommendations for adults and children, based on 
exhaustive literature review, for the managing of PONV and the definition of risk groups ${ }^{21}$. Chart I shows risk factors for adults and children.

\section{PONV PREVENTION AND TREATMENT}

Several questions are posed when the issue PONV prevention versus treatment is addressed. Should we prevent it, or should we wait for its development and then treat. If PONV is preventively treated which drugs or doses should be used? If deciding for a conservative approach and treating after the development of PONV, which drugs or doses should be used? If patients are preventively treated and even then develop PONV, which drugs or doses should be used?

Chart I - Risk Factors for PONV in Adults and Children (b) (Gan et al. ${ }^{21}$ )

\begin{tabular}{|c|c|}
\hline \multicolumn{2}{|c|}{$\begin{array}{l}\text { "Consensus for PONV Management" } \\
\text { Risk Factors for Adults }\end{array}$} \\
\hline \multirow[t]{3}{*}{ Patients } & Female gender \\
\hline & Non smoker \\
\hline & History of motion sickness \\
\hline \multirow[t]{3}{*}{ Anesthesia } & Inhalational anesthetics \\
\hline & $\mathrm{N}_{2} \mathrm{O}$ \\
\hline & Intra and postoperative opióides \\
\hline \multirow[t]{2}{*}{ Surgery } & $\begin{array}{l}\text { Duration: above } 30 \text { minutes increases } \\
\text { risk in } 60 \%\end{array}$ \\
\hline & $\begin{array}{l}\text { Types of surgery: celioscopy, ENT, } \\
\text { neurosurgery, breast, strabismus, } \\
\text { laparotomy, plastic surgery }\end{array}$ \\
\hline \multicolumn{2}{|c|}{$\begin{array}{l}\text { "Consensus for PONV Management" } \\
\text { Risk Factors for Children }\end{array}$} \\
\hline \multicolumn{2}{|c|}{$\begin{array}{l}\text { Risk factors for children are similar to adults, with some uniquenesses: } \\
\text { - Pediatric studies are limited to data on vomiting, not on nausea; } \\
\text { - The incidence of vomiting in children is twice the incidence in adults; } \\
\text { - The difference in gender is not observed with children; } \\
\text { - Risk is more consistently increased with certain types of surgery. }\end{array}$} \\
\hline
\end{tabular}

With preventive treatment, many patients at low risk for PONV will be unnecessarily treated, in addition to being exposed to the adverse effects of drugs, while other patients will present PONV despite of the treatment. Routine antiemetics are associated to high costs, which in a time of costs and resources contention should be avoided. However, preventive antiemetics in moderate to high-risk patients have an effective cost-benefit ratio and are associated to higher level of patients' satisfaction ${ }^{22}$.

The 2003 consensus conference has recommended preventive treatment for patients at moderate to high risk of PONV or for those with potential morbidity associated to PONV, specifically suture dehiscence, esophageal rupture, hematoma and aspiration pneumonia ${ }^{21}$. Whenever possible, it is recommended to identify risk factors and minimize them. Visser et al. have shown that propofol for anesthetic induction and maintenance decreases the incidence of early PONV (first 6 postoperative hours) ${ }^{23}$. The incidence of PONV decreases when inhalational anesthetics or nitrous oxide are avoided ${ }^{24}$. Neostigmine in doses equal to or below $2.5 \mathrm{mg}$ decreases PONV ${ }^{18}$. Sinclair et al. has shown that loco-regional anesthesia decreases PONV risk 11 times as compared to general anesthesia ${ }^{12}$. Yogendran et al. has concluded that effective fluid therapy with blood pressure maintenance during induction decreases the incidence of PONV ${ }^{25}$.

High perioperative oxygen inspired fractions decreases in $50 \%$ the incidence of nausea and vomiting ${ }^{26}$. Last but not least, less opioids and effective analgesia (surgical wound infiltration with local anesthetics or cycloxygenase inhibitor administration) also contribute to decrease the incidence of nausea and vomiting ${ }^{26}$.

\section{ANTIEMETIC DRUGS USED FOR PONV PREVENTIVE TREATMENT (Table II)}

Serotonin receptor antagonists: there is no difference in efficacy and safety profile within this group of drugs ondansetron, dolasetron, granisetron or etropisetron ${ }^{21}$. These are more effective when administered at surgery completion and are more useful to prevent vomiting as compared to nausea ${ }^{27,28}$.

Dexamethasone: the action mechanism of steroids as antiemetic drugs is still to be explained. They are more effective when administered before anesthetic induction. Most popular intravenous dose is 8 to $10 \mathrm{mg}$, but lower doses $(2.5$ to $5 \mathrm{mg}$ ) are also effective. Notwithstanding side effects of these drugs, such as surgical wound infection or adrenal gland suppression, no side effects were observed after bolus doses $^{29}$.

Droperidol: is one of the most widely studied antiemetics to prevent PONV and is similar in efficacy to ondansetron ${ }^{30}$. It is better to prevent nausea than to prevent vomiting and is more effective when administered at surgery completion ${ }^{31}$. In morphine patient-controlled analgesia this seems to be the most effective antiemetic drug. Doses between 15 and $50 \mu \mathrm{g}$ droperidol per mg of morphine are those with best efficacy/side effects ratio ${ }^{32}$. In 2001, the Food and Drug Administration has published a warning about the association of droperidol in patients with prolonged QT interval at ECG and/or Torsade de Pointes, which in some cases has resulted in arrhythmias and death.

This warning was based on 10 clinical cases observed along 30 years of clinical use, in which doses equal to or below 1.25 mg were used ${ }^{33}$. As a consequence, the laboratory producing droperidol has recommended ECG monitoring during and 2 to 3 hours after its administration and has counterindicated it for male patients with QT intervals above $440 \mathrm{~ms}$ or for female patients with QT intervals above $450 \mathrm{~ms}$. Other risk patients are those with congestive heart failure, bradycardia, ventricular hypertrophy, hypokalemia, hypomagnesemia, patients medicated with diuretics or other drugs which may originate prolonged QT interval. Some droperidol advocates argue that there are no descriptions in indexed publications 
Table II - Antiemetic Doses for Adults and Children (Gan et al. ${ }^{21}$ )

\begin{tabular}{|c|c|c|c|}
\hline Administration & Drug & Adult Dose & Children Dose \\
\hline Before induction & Dexamethasone & $\begin{array}{l}5 \text { to } 10 \mathrm{mg} \\
\text { (intravenous) }\end{array}$ & $\begin{array}{c}150 \mu \mathrm{gg} \cdot \mathrm{kg}^{-1} \\
(\text { maximum } 8 \mathrm{mg} \text { ) }\end{array}$ \\
\hline \multirow[t]{5}{*}{ After surgery } & Dolasetron & $\begin{array}{l}12.5 \mathrm{mg} \\
\text { (intravenous) }\end{array}$ & $\begin{array}{c}350 \mu \mathrm{g} \cdot \mathrm{kg}^{-1} \\
\text { (maximum } 12.5 \mathrm{mg} \text { ) }\end{array}$ \\
\hline & Droperidol & $\begin{array}{c}0.625 \text { to } 1,25 \mathrm{mg} \\
\text { (intravenous) }\end{array}$ & $\begin{array}{c}50 \text { to } 75 \mu \mathrm{g} . \mathrm{kg}^{-1} \\
\text { (maximum } 1.25 \mathrm{mg} \text { ) }\end{array}$ \\
\hline & Granisetron & $\begin{array}{l}0.35 \text { to } 1 \mathrm{mg} \\
\text { (intravenous) }\end{array}$ & \\
\hline & Ondansetron & $\begin{array}{l}4 \text { to } 8 \mathrm{mg} \\
\text { (intravenous) }\end{array}$ & $\begin{array}{l}50 \text { to } 100 \mu \mathrm{g} \cdot \mathrm{kg}^{-1} \\
\text { (maximum } 4 \mathrm{mg} \text { ) }\end{array}$ \\
\hline & Tropiseton & $\begin{array}{l}5 \mathrm{mg} \\
\text { (intravenous) }\end{array}$ & \\
\hline $\begin{array}{l}\text { Night before surgery or } \\
4 \text { hours before surgery completion }\end{array}$ & Scopolamine & Transcutaneous patch & \\
\hline Without indication & Dimenhirinate & $\begin{array}{c}1 \text { to } 2 \text { mg.kg- } \\
\text { (intravenous } \\
0.5 \mathrm{mg} \cdot \mathrm{kg}^{-1} \\
\text { (intramuscular) }\end{array}$ & $0.5 \mathrm{mg} \cdot \mathrm{kg}^{-1}$ \\
\hline
\end{tabular}

of clinical cases in which prolonged QT interval, arrhythmias or death were associated to droperidol in adequate doses to prevent PONV.

Other antiemetic drugs: subcutaneous scopolamine should be administered the night before surgery or approximately 4 hours before surgery completion ${ }^{34}$. It is not recommended for the elderly and its limitation is late onset ( 2 to 4 hours after administration) ${ }^{35}$. Phenothyazides (prometazine and proclhorperazine) are effective if administered at surgery completion, but their adverse effects (sedation, dizziness and dehydration) limit its use, especially in outpatient regimen ${ }^{36}$. Intramuscular ephedrine seems to be effective, especially if nausea and vomiting are associated to dehydration or hypotension ${ }^{37}$.

Non-pharmacological techniques: acupuncture, compression, intradermal nervous stimulation and hypnosis were effective if used before surgery ${ }^{38}$, but they lack further evaluation.

Insufficient Evidences: intravenous metoclopramide in doses used in the clinical practice $(10 \mathrm{mg})$ is not recommended to treat PONV ${ }^{39}$, as well as ginger root ${ }^{40}$ or cannabinoids ${ }^{41}$.

A multimodal antiemetic approach has been recommended in recent years, based on the multifactorial concept of PONV etiology. So, using more than one class of antiemetics it would be possible to reach more than one receptor involved in PONV.

The already mentioned consensus conference has recommended no preventive treatment for low risk patients; for moderate risk patients, prevention with single or combined therapy; for high risk patients, prevention with 3 antiemetic drugs of different classes ${ }^{21}$. However, a recent study has stressed that the combination of different therapies is associated to higher costs, higher risk of side effects and lack of cumulative benefit of several antiemetic drugs. For these au- thors, combined therapy should be kept for patients at high risk for PONV or those who may be at risk due to PONV ${ }^{42}$. This specific group has a higher number of candidates to antiemetic prevention, since the incidence of PONV is two-fold. First line drugs should be $5-\mathrm{HT}^{3}$ receptor antagonists. Due to its extra-pyramidal effects, droperidol should be kept for admitted children when all other therapies have failed ${ }^{21}$.

Guidelines for the treatment of PONV of patients who have not received preventive treatment or those for whom prevention has failed (Chart II) ${ }^{21}$.

Chart II - PONV Treatment in Patients not Receiving Preventive Treatment or in those for whom it Failed (Gan et al. ${ }^{21}$ )

If initial therapy Treatment

No prevention or dexamethasone

Decreased serotoninergic receptor antagonist doses

Serotoninergic receptor antagonists and a $2^{\text {nd }}$ drug of different class

Triple therapy with serotoninergic receptor antagonists and $2^{\text {nd }}$ and

$3^{\text {rd }}$ drugs of different classes

Different class drug

If $\mathrm{PONV}$ is present $<6$ postoperative hours: different class of drug or $20 \mathrm{mg}$ propofol (if UCPO)

If $P O N V$ is present $\geq 6$ postoperative hours: repeat serotoninergic receptor antagonists and droperidol or a different class of drug

First, all drugs or mechanical factors that may originate PONV should be withdrawn and then treatment should be started.

Patients not receiving preventive PONV treatment should be given $5-\mathrm{HT}_{3}$ antagonists. One fourth of preventive doses have shown to be effective. There are still few data on doses and efficacy of other antiemetics to treat PONV. In patients with failed preventive treatment, low $5-\mathrm{HT}_{3}$ receptor antagonist doses are recommended. 
If prevention with $5-\mathrm{HT}_{3}$ receptor antagonists has failed, subsequent doses of the same class of drugs should not be used during the first 6 postoperative hours. If prevention with $5-\mathrm{HT}_{3}$ receptor antagonists associated to dexamethasone was ineffective, a different class of antiemetics should be administered.

In general, patients with PONV in the first 6 postoperative hours should be treated with different classes of antiemetics than those used for prevention. If PONV is present 6 hours after surgery, drugs used for prevention may be repeated, with the exception of scopolamine or dexamethasone, because they act for more than 6 hours.

Low propofol doses may be used, provided patients are under continuous surveillance.

\section{CONCLUSION}

Although PONV management has improved in recent years, it is still frequent in high-risk groups.

The identification of risk factors for PONV, frequently done during preoperative evaluation, helps preventive therapy. The publication in 2003 of international recommendations for PONV management allowed the definition of patients benefiting from single, double or triple therapy as a function of identified risk level.

From different available drugs, droperidol, $5 \mathrm{HT}_{3}$ receptor antagonists and dexamethasone play a distinctive role in controlling PONV, and their association, based on action mechanisms and efficacy/tolerance ratio, has allowed for more effective PONV control.

Notwithstanding limited data in the literature and variations in the identification of risk factors, recommendations for adults are extrapolated to children. It should be reminded that these recommendations do not rule out the individualized analysis and its adaptation to populations, surgical and anesthetic techniques.

\section{REFERÊNCIAS - REFERENCES}

01. Bonica J, Crepps W, Monk B et al - Postanesthetic nausea, retching and vomiting; evaluation of cyclizine (marezine) suppositories for treatment. Anesthesiology, 1958;19:532-540.

02. Apfel CC, Laara E, Koivuranta M et al - A simplified risk score for predicting postoperative nausea and vomiting: conclusions from cross-validations between two centers. Anesthesiology, 1999;91:693-700.

03. Leman J - Surgical and patient factors involved in postoperative nausea and vomiting. $\mathrm{Br} J$ Anaesth, 1992;69:(7Suppl1): 24S-32S.

04. Gold BS, Kitz DS, Lecky JH et al - Unanticipated admission to the hospital following ambulatory surgery. JAMA, 1989;262: 3008-3010.

05. Eberhart LH, Morin AM, Wulf $\mathrm{H}$ et al - Patient preferences for immediate postoperative recovery. $\mathrm{Br} \mathrm{J}$ Anaesth, 2002;89: 760-761.

06. Macario A, Weinger M, Carney S et al - Which clinical anesthesia outcomes are important to avoid ? The perspective of patients. Anesth Analg, 1999;89:652-658.
07. Gan T, Sloan F, Dear Gde L et al - How much are patients willing to pay to avoid postoperative nausea and vomiting? Anesth Analg, 2001;92:393-400.

08. Tramer MR - A rational approach to the control of postoperative nausea and vomiting: evidence from systematic reviews. Acta Anaesthesiol Scand, 2001;45:4-13.

09. Watcha MF, White PF - Postoperative nausea and vomiting. Its etiology, treatment, and prevention. Anesthesiology, 1992;77: 164-184.

10. Junger A, Hartmann B, Benson M et al - The use of an anesthesia information management system for prediction of antiemetic rescue treatment at the postanesthesia care unit. Anesth Analg, 2001:92:1203-1209.

11. Koivuranta M, Laara E, Snare L et al - A survey of postoperative nausea and vomiting. Anaesthesia, 1997;52:443-449.

12. Sinclair PR, Chung F, Mezei G - Can postoperative nausea and vomiting be predicted? Anesthesiology, 1999;91:109-118.

13. Thomas $R$ - The effect of smoking on postoperative nausea and vomiting. Anaesthesia, 2000;55:1032-1033.

14. Apfel CC, Kranke P, Eberhart LH et al - Comparison of predictive models for postoperative nausea and vomiting. $\mathrm{Br} \mathrm{J}$ Anaesth, 2002;88:234-240.

15. Pierre S, Benais H, Pouymayou J - Apfel's simplified score may favourably predict the risk of postoperative nausea and vomiting. Can J Anaesth, 2002;49:237-242.

16. Scuderi PE, James RL, Harris $L$ et al - Multimodal antiemetic management prevents early postoperative vomiting after outpatient laparoscopy. Anesth Analg, 2000;91:1408-1414.

17. Greif R, Laciny S. Rapf B et al - Supplemental oxygen reduces the incidence of postoperative nausea and vomiting. Anesthesiology, 1999;91:1246-1252.

18. Tramer MR, Fuchs-Buder T - Omitting reversal of neuromuscular block: effect on postoperative nausea and vomiting and risk of residual paralysis. A systematic review. $\mathrm{Br} \mathrm{J}$ Anaesth, 1999;82:379-386.

19. Apfel CC, Greim CA, Haubitz I et al - A risk score to predict the probability of postoperative vomiting in adults. Acta Anaesthesiol Scand, 1998;42:495-501.

20. Thomas R, Jones NA, Strike P - The value of risk scores for predicting postoperative nausea and vomiting when used to compare patient group in randomised controlled trial. Anaesthesia, 2002; $57: 1119-1128$

21. Gan TJ, Meyer T, Apfel CC et al - Consensus guidelines for managing postoperative nausea and vomiting. Anesth Analg, 2003;97:62-71.

22. Hill RP, Lubarsky DA, Phillips-Bute B et al - Cost-effectiveness of prophylactic antiemetic therapy with ondansetron, droperidol, or placebo. Anesthesiology, 2000;92:958-967.

23. Visser K, Hassink EA, Bonsel GJ et al - Randomized controlled trial of total intravenous anesthesia with propofol versus inhalation anesthesia with isoflurane - nitrous oxide: postoperative nausea with vomiting and economic analysis. Anesthesiology, 2001;95:616-626.

24. Apfel CC, Kranke P, Katz MH et al - Volatile anaesthetics may be main cause of early but not delayed postoperative vomiting: a randomized controlled trial of factorial design. $\mathrm{Br} \mathrm{J}$ Anaesth, 2002;88:659-668

25. Yogendran S, Asokumar B, Cheng DC et al - A prospective, randomized, double-blinded study of the effect of intravenous fluid therapy on adverse outcomes on outpatient surgery. Anesth Analg, 1995;80:682-686.

26. Apfel CC, Kranke P, Eberhart LH et al - Comparison of predictive models for postoperative nausea and vomiting. $\mathrm{Br} \mathrm{J}$ Anaesth, 2002;88:234-240. 
27. Tramer MR, Reynolds DJ, Moore RA et al - Efficacy, dose-response, and safety of ondansetron in prevention of postoperative nausea and vomiting: a qualitative systematic review of randomized placebo-controlled trials. Anesthesiology, 1997;87:1277-1289.

28. Sun $\mathrm{R}$, Klein KW, White PF - The effect of timing of ondansetron administration in outpatient undergoing otolaryngologic surgery. Anesth Analg, 1997;84:331-336.

29. Henzi I, Walder B, Tramer MR - Dexamethasone for the prevention of postoperative nausea and vomiting: a quantitative systematic review. Anesth Analg, 2000;90:186-194.

30. Millo J, Siddons M, Innes R et al - Randomised double-blind comparison of ondansetron and droperidol to prevent postoperative nausea and vomiting associated with patient-controlled analgesia. Anaesthesia, 2001;56:60-65.

31. Henzi I, Sonderegger J, Tramer MR - Efficacy, dose-response, and adverse effects of droperidol for prevention of postoperative nausea and vomiting. Can J Anaesth, 2000;47:537-551.

32. Culebras X, Corpataux JB, Gaggero G et al - The antiemetic efficacy of droperidol added to morphine patient-controlled analgesia: a randomized, controlled, multicenter dose-finding study. Anesth Analg, 2003;97:816-821.

33. Habib AS, Gan TJ - Food and drug administration black box warming on the perioperative use of droperidol: a review of the cases. Anesth Analg, 2003;96:1377-1379.

34. Kranke P, Morin AM, Roewer N et al - The efficacy and safety of transdermal scopolamine for the prevention of postoperative nausea and vomiting: a quantitative systematic review. Anesth Analg, 2002;95:133-143.

35. Bailey PL, Streisand JB, Pace NL et al - Transdermal scopolamine reduces nausea and vomiting after outpatient laparoscopy. Anesthesiology, 1990;72:977-980.

36. Chen JJ, Frame DG, White TJ - Efficacy of ondansetron and prochlorperazine for the prevention of postoperative nausea and vomiting after total hip replacement or total knee replacement procedures: a randomized, double-blind, comparative trial. Arch Intern Med, 1998;158:2124-2128.

37. Hagemann E, Halvorsen A, Holgersen O et al - Intramuscular ephedrine reduces emesis during the first three hours after abdominal hysterectomy. Acta Anaesthesiol Scand, 2000;44: 107-111.

38. Lee A, Done ML - The use of nonpharmacologic techniques to prevent postoperative nausea and vomiting: a meta-analysis. Anesth Analg, 1999;88:1362-1369.
39. Henzi I, Walder B, Tramer MR - Metoclopramide in the prevention of postoperative nausea and vomiting: a quantitative systematic review of randomized placebo-controlled studies. $\mathrm{Br} \mathrm{J}$ Anaesth, 1999;83:761-771.

40. Ernst E, Pittler MH - Efficacy of ginger for nausea and vomiting: a systematic review of randomized clinical trials. $\mathrm{Br} \mathrm{J}$ Anaesth, 2000;84:367-371.

41. Tramer MR, Carroll D, Campbell FA et al - Cannabinoids for control of chemotherapy-induced nausea and vomiting: quantitative systemic review. BMJ, 2001;323:16-21.

42. Apfel CC, Korttila K, Abdalla M et al - A factorial trial of six interventions for the prevention of postoperative nausea and vomiting. N Engl J Med, 2004;350:2441-2451.

\section{RESUMEN}

Lages N, Fonseca C, Neves A, Landeiro N, Abelha FJ - Náuseas y Vómitos en el Posoperatorio: Una Revisión del "Pequeño-Grande" Problema

JUSTIFICATIVA Y OBJETIVOS: A pesar de la averiguación continuada y del desarrollo de nuevos fármacos y técnicas, las náuseas y vómitos en el posoperatorio (NVPO) son frecuentes y pueden aportar para el desarrollo de complicaciones con consecuente aumento de los costeos hospitalarios y de los recursos humanos. Los objetivos de este artículo son la revisión de los mecanismos fisiológicos, de los factores de riesgo y las medidas terapéuticas disponibles para el manoseo de NVPO.

CONTENIDO: Varias son las estrategias de manoseo de NVPO sugeridas en este artículo, destacándose, sin embargo, las líneas de orientación emitidas por Gan en 2003. Éstas, constituyen la contribución más reciente para la estratificación del riesgo, prevención y tratamiento de los pacientes con NVPO

CONCLUSIONES: Aunque el manoseo del NVPO haya mejorado en los últimos años, éstos aún ocurren frecuentemente en grupos de riesgo elevado. La estrategia actual para la prevención y manoseo de NVPO permanece por establecer y las líneas de orientación de Gan deberán ser adaptadas a cada población de pacientes y a la institución hospitalaria. 